\title{
Casimir force between bimetallic heterostructures
}

\author{
J. Bárcenas, L. Reyes, and R. Esquivel Sirvent* \\ Instituto de Física, Universidad Nacional Autónoma de México, Apartado Postal 20-364 \\ Ciudad Universitaria, México, 01000, México
}

Received 19 July 2004, revised 21 July 2004, accepted 11 August 2004

Published online 9 May 2005

PACS 12.20.Ds, 42.50.Lc, 78.20.-e

We present a general method for calculating the Casimir force between heterostructures using an effective surface impedance approach. Within this formalism we study the effect of thin film coatings on the force. As a case study we present results for a system made of alternate layers of $\mathrm{Mg}$ and $\mathrm{Ni}$ and evaluate the effect that Pd coatings have on the Casimir force.

(C) 2005 WILEY-VCH Verlag GmbH \& Co. KGaA, Weinheim

1 Introduction The Casimir effect is a very short range force that results from the modification of the zero point electromagnetic field oscillations when physical boundaries modify the quantum vacuum fluctuations. For example, two neutral parallel plates made of a perfect conductor and separated a distance $L$ will attract each other with a force given by [1]

$$
F_{0}=-\frac{\hbar c \pi^{2}}{240 L^{4}}
$$

With the recent growth of nano physics, the Casimir effect has gained more attention, and this time also on the experimental side [2-6], because it is clear that Casimir forces will have to be taken into account where micro and nanostructures are involved $[7,8]$. These experiments were made between a sphere and a plate, a setup that has proved more easily applicable in experiments. Recently Ianuzzi et al. [9] presented precise measurements of the Casimir force using microtorsional devices. The goals of their investigations are quite significant, for they used an exotic material (hydrogen switchable mirrors (HSM)) to probe how optical properties may change the Casimir force and thereby allow to fine-tune it, all this in sights of controlling the force in nanostructures. The results were not quite as expected (the use of HSM turned out not to modify the Casimir force significantly) although other questions arose such as the role of Pd in the Casimir attraction between HSM's.

The possible applications of the Casimir force in nanodevices depend on how the force can be modified in a given system. In previous works the magnitude of Casimir forces was altered using heterostructures and thermally activated materials. These calculations were restricted to the one-dimensional case $[10,11]$.

In this paper we present a calculation for the Casimir force between bimetallic superlattices in the 3D case and study the effect that Pd coatings. Our approach is based on the concept of effective surface impedance.

2 Theory The Casimir effect results from the quantum vacuum fluctuations that produces photons of all frequencies in the absence of boundaries. When boundaries are present only photons that satisfy the boundary conditions are allowed. Let the wave vector associated to any of these photons be $\vec{q}=(Q, 0, K)$, such that $|\vec{q}|=\omega / c$.

\footnotetext{
* Corresponding author e-mail: raul@ sica.unam.mx
} 
Consider now two different parallel slabs (labelled 1 and 2) separated a distance $L$ as shown in the inset of Figure 1. Each slab can be homogeneous or inhomogeneous (it can be a superlattice, for example). The force can be expressed in terms of the reflectivities of the slabs as $[12,13]$

$$
\frac{F(L)}{A}=\frac{\hbar c}{2 \pi^{2}} \int_{0}^{\infty} d Q Q \int_{q \geq 0} d k \frac{k^{3}}{q} \operatorname{Re} \frac{1}{k}\left[\frac{1}{\xi^{s}-1}+\frac{1}{\xi^{p}-1}\right],
$$

where $\xi^{s, p}=\left(r_{1}^{s, p} r_{2}^{s, p} \exp (2 i k L)\right)^{-1}$, and $r_{1,2}^{s, p}$ is the reflectivity from each layer for $s$ and $p$ polarized waves. In the limit of a perfect conductor, Equation (2) reduces to Eq. (1) and when the reflectivities are replaced by the Fresnel coefficients the so called Lifshitz formula for semi-infinite homogeneous media is obtained.

As compared to other procedures [14], Eq. (2) allows for a direct calculation of the Casimir force provided the reflectivities are known without having to make use of the fluctuation-dissipation theorem or getting into the problem of quantizing the electromagnetic field in dissipative media. In the case of bimetallic superlattices the optical response is well studied [15] and in this paper we just summarize the procedure for obtaining the reflectivities. We assume a bimetallic superlattice made of two alternate layers ( $a$ and $b$ ) each characterized by its local dielectric function $\epsilon_{a, b}(\omega)$ and its thickness $d_{a, b}$. Each bilayer (or unit cell) is characterized by a transfer matrix $M_{u}^{s, p}$ constructed ad hoc for each wave polarization $p, s$. The reflectivities can be written in terms of the impedances as

$$
\begin{aligned}
& r_{a}^{s}=\frac{Z^{s}-Z_{0}^{s}}{Z^{s}+Z_{0}^{s}} \\
& r_{a}^{p}=\frac{Z_{0}^{p}-Z^{p}}{Z_{0}^{p}+Z^{p}},
\end{aligned}
$$

where the subindex 0 refers to vacuum and $Z_{0}^{p}=k / q, Z_{0}^{s}=q / k$ and $Z^{p, s}$ is the ratio of the tangential components of the electric and magnetic field for each polarization, evaluated at the surface of the superlattice. If the superlattice consists of $n$ bilayers the effective surface impedances are given by

$$
Z^{p, s}=(-1)_{s} \frac{M_{22} Z_{0}^{p, s}-M_{12}}{M_{11}-M_{21} Z_{0}^{p, s}}
$$

In the last equation we define $M=M_{u}^{n}$ as the $n^{t h}$ power of the transfer matrix of one bilayer and the minus sign corresponds to $s$ polarized waves.

For a semi-infinite system we can use Bloch's theorem to find the effective surface impedances as

$$
Z^{p, s}(0)=-\frac{M_{12}^{s, p}}{M_{11}^{p, s}-\exp \left(i \kappa^{p, s} d\right)},
$$

where $\kappa^{p, s}$ is the one dimensional Bloch wave vector that satisfies the equation $2 \cos \left(\kappa^{p, s} d\right)=\operatorname{Tr} M_{u}^{p, s}$, being $d=d_{a}+d_{b}$ the thickness of the unit cell.

3 Case study. Mg/Ni heterostructures To study how a layered system can change the Casimir force we consider first the force between two equal hetersotructures made of alternate layers of $\mathrm{Mg}$ and $\mathrm{Ni}$, assuming that the boundary between both materials is well defined. This system coated with $\mathrm{Pd}$ is an example of a hydrogen switchable mirror as discussed by Richardson [16] and used by Iannuzzi in recent measurements of Casimir forces [9]. The local dielectric function for each of the materials is modelled using a Drude model where the plasma frequency and damping is taken from [17]. In all the calculation we use a structure with $n=7$ bilayers and the Mg layer is $100 \AA$ thick while the Ni layer is $20 \AA$. The force between these two heterostructures is $F$. 
Since we want to study the effect of Pd layers, we also calculate the force when the heterostructure is coated with a Pd layer. This force is denoted by $F_{p}$. In this case Eq. (4) is valid considering that the total transfer matrix of the system is $M^{s, p}=M_{u}^{s, p} M_{P d}^{s, p}$, where $M_{P d}^{s, p}$ is the transfer matrix for the Pd film. To see the effect of a Pd coating, in Figure 1 we plot $\Delta=\left(F-F_{p}\right) / F$ as a function of the separation $L$. This is a measure of the difference on the force with and without the Pd. As seen in Fig. 1 as the thickness of the Pd layer increases so does $\Delta$ at a fixed separation $L$. For comparison, we have also calculated the Casimir force between two half-spaces made of Pd. Another system of interest is the Casimir force between a

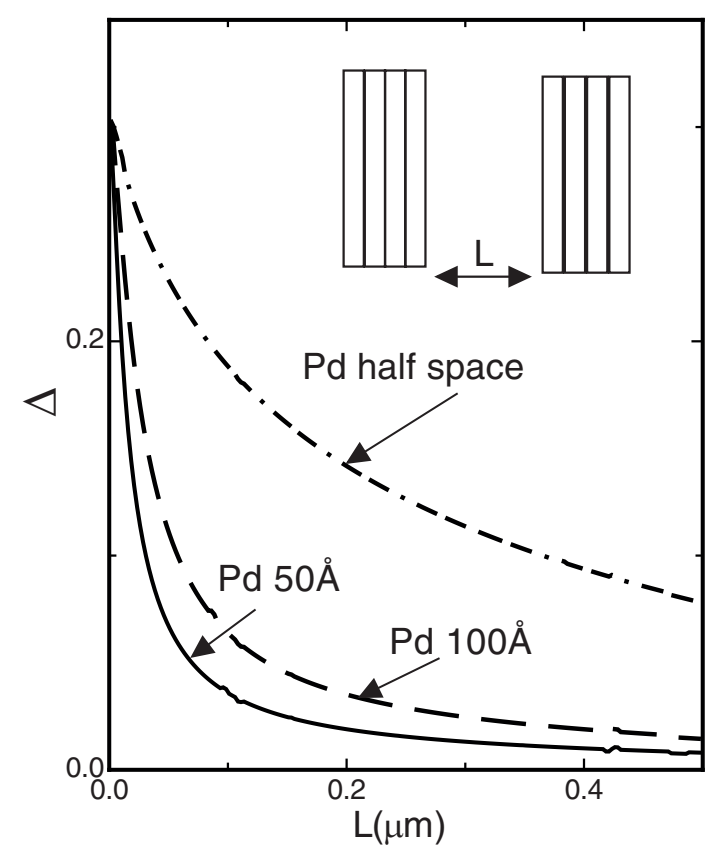

Fig. 1 Difference between the Casimir force between two $\mathrm{Mg} / \mathrm{Ni}$ layered structures with and without Pd. Since $\Delta$ is positive as a function of the separation $L$, the effect of the Pd is to reduce the magnitude of the force. The inset shows the configuration of the system.

$\mathrm{Mg} / \mathrm{Ni}$ heterostructure and a Au half space (see inset in Fig. 2). This is similar to the geometry used in the experiments by Iannuzzi et al. [9]. Again, we calculate the force with and without $\operatorname{Pd}$ and plot $\Delta$ as a function of the separation. This is shown in Figure 2. In this case the Pd coating is $20 \AA$ thick. In this case, also for small separations the difference due to the Pd coating is significant and in the curve a small kink can be observed when the separation is $L \sim 0.11 \mu \mathrm{m}$. This corresponds to the plasma wavelength of Pd, suggesting a resonant effect due to the thin film.

4 Conclusions We presented a 3-D calculation of the the Casimir force between bimetallic heterostructures and study the effect that coating layers can have. The calculations are based on the concept of effective surface impedance, long used in the field of optical properties of superlattices. However, it provides a straightforward method for the computation of Casimir forces as compared to other techniques described in the literature [14]. As case study we consider an heterostructure made of $\mathrm{Mg}$ and $\mathrm{Ni}$ and study the effect of Pd coatings. This system is similar to the ones used in recent Casimir experiments. The effect of the Pd coatings is significant and the force decreases with the coating. In future work, the influence of surface plasmon-polariton coupling between the thin films and its influence on the Casimir force will be considered. 


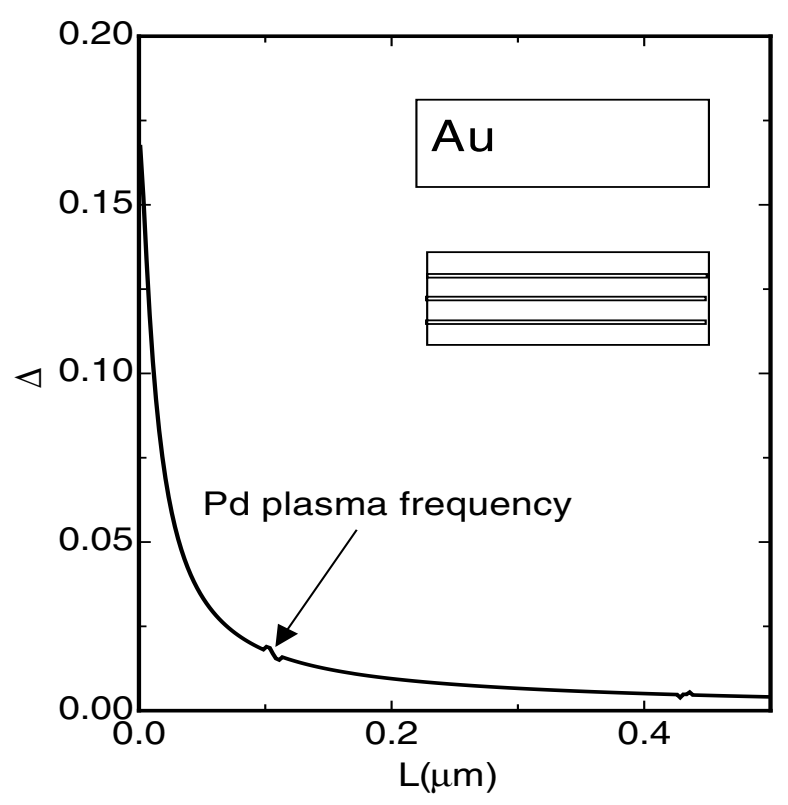

Fig. 2 Difference between the Casimir force between a Au half-space and a $\mathrm{Mg} / \mathrm{Ni}$ layered structure with and without Pd. The Pd layer is $20 \AA$ thick.

Acknowledgements Partial support from CONACyT Grant 36651-E and DGAPA-UNAM Grant 116002 and IFUNAM . We thank W.L. Mochán and C. Villarreal for helpful discussions.

\section{References}

[1] H. B. G. Casimir, Proc. Kon. Ned. Akad. Wet. 51, 793 (1948).

[2] S. K. Lamoreaux, Phys. Rev. Lett. 78, 5 (1997).

[3] U. Mohideen and A. Roy, Phys. Rev. Lett. 81, 4549 (1998).

[4] B. W. Harris, F. Chen, and U. Mohideen, Phys. Rev. A 62, 052109 (2000).

[5] H. B. Chan, V. A. Aksyuk, R. N. Kliman, D. J. Bishop, and F. Capasso, Science 291, 1942 (2001).

[6] R. Decca, D. López, E. Fischbach, and D. E. Krause, Phys. Rev. Lett. 91, 050402 (2003).

[7] M. Serry, D. Walliser, and J. Maclay, J. Appl. Phys. 84, 2501 (1998).

[8] J. Maclay, J. Hammer, Vacuum forces in microcavities, 7th International Conference on Squeezed States and Uncertainty Relations, Boston, MA, June 4-6, (2001).

[9] D. Ianuzzi, M. Lisanti, and F. Capasso, Proc. Natl. Acad. Sci. 101, 4019 (2004).

[10] R. Esquivel-Sirvent, C. Villarreal, and G. H. Cocoletzi, Phys. Rev. A 64, 052108 (2001).

[11] R. Esquivel-Sirvent and C. Villarreal y C. Noguez, Mater. Res. Soc. Symp. 703, V.3.3.1 (2002).

[12] W.L. Mochán, C. Villareal, and R. Esquivel-Sirvent, Rev. Mex. Fis. 48, 339 (2002).

[13] R. Esquivel-Sirvent, C. Villarreal, and W. M. Mochan,Phys. Rev. A 68, 052103 (2003).

[14] C. Raabe, L. Knoll, and D. G. Welsch, Phys. Rev. A 68, 033810 (2003).

[15] E. López Olazagastegui, G. H. Cocoletzi, and W. L. Mochán, Solid State Commun. 78, 9 (1991).

[16] T. J. Richardson, J. L. Slack, R. D. Armitage et al., Appl. Phys. Lett. 78, 3047 (2001).

[17] M. A. Ordal, R. J. Bell, R. W. ALexander, L.L. Long, and M. R. Querry, Appl. Opt. 24, 4493 (1985). 